\title{
Clavien-Dindo Classification of Surgical Complications
}

National Cancer Institute

\section{Source}

National Cancer Institute. Clavien-Dindo Classification of Surgical Complications. NCI Thesaurus. Code C133758.

A classification system for post-surgical complications based on the type of therapy needed to correct the complication, consisting of a 5-grade rating scale. 